\title{
Threonine biosynthetic genes are essential in Cryptococcus neoformans
}

Correspondence

John H. McCusker

mccus001@mc.duke.edu

Received 16 April 2008

Revised 20 May 2008

Accepted 27 May 2008

\author{
Joanne M. Kingsbury and John H. McCusker
}

Department of Molecular Genetics and Microbiology, Box 3020, Duke University Medical Center, Durham, NC 27710, USA

\begin{abstract}
We identified and attempted to disrupt the Cryptococcus neoformans homoserine and/or threonine biosynthetic genes encoding aspartate kinase (HOM3), homoserine kinase (THR1) and threonine synthase (THR4); however, each gene proved recalcitrant to disruption. By replacing the endogenous promoters of HOM3 and THR1 with the copper-repressible CTR4-1 promoter we showed that HOM3 and THR1 were essential for the growth of $C$. neoformans in rich media, when ammonium was the nitrogen source, or when threonine was supplied as an amino acid instead of a dipeptide. Moreover, the severity of the growth defect associated with HOM3 or THR1 repression increased with increasing incubation temperature. We believe this to be the first demonstration of threonine biosynthetic genes being essential in a fungus. The necessity of these genes for C. neoformans growth, particularly at physiologically relevant temperatures, makes threonine biosynthetic genes ideal anti-cryptococcal drug targets.
\end{abstract}

\section{INTRODUCTION}

Amino acid biosynthetic pathways provide attractive candidates for antifungal drug targets since many of these pathways are conserved throughout the fungi and are absent from humans. One such pathway of interest is the threonine biosynthetic pathway, in which threonine is produced from aspartate in five enzymic steps via the intermediate homoserine, which is also required for methionine synthesis (Fig. 1; reviewed by Jones \& Fink 1982). In the yeast Saccharomyces cerevisiae, this pathway is regulated at the level of transcription by general control (Hinnebusch, 1992; Mountain et al., 1991), and of enzyme activity, particularly by threonine feedback inhibition of aspartate kinase (Hom3p) at the initial step of the pathway (MartinRendon et al., 1993; Ramos \& Calderon, 1992). In addition to auxotrophy, a number of deleterious phenotypes have been attributed to threonine biosynthetic mutants (ArevaloRodriguez et al., 2004; Birrell et al., 2001, 2002; Care et al., 2004; Deutschbauer et al., 2002; Dunn et al., 2006; Enyenihi \& Saunders, 2003; Giaever et al., 2002; Roberg et al., 1997), some defects of which, such as temperature sensitivity, salt sensitivity and being petite-negative, could also influence fungal survival in vivo. Moreover, the threonine-biosynthetic intermediate homoserine is also required for biosynthesis of methionine, itself a central metabolite, and threonine is required for isoleucine biosynthesis. Significantly, we and others have shown that various fungal methionine (Met2p, Met3p and Met6p) and isoleucine (Ilv2p), as well as

Abbreviation: BCS, bathocuproinedisulfonic acid.

A supplementary table of primers is available with the online version of this paper. threonine (Hom3p), biosynthetic enzymes are required for fungal survival in vivo and/or virulence (Kingsbury et al., 2004a, 2006; Nazi et al., 2007; Pascon et al., 2004; Yang et al., 2002).

Amino acid auxotrophy has been shown to be particularly deleterious in the human-pathogenic fungus Cryptococcus neoformans. Compared with $S$. cerevisiae, various auxotrophies are less well supplemented by the amino acids for which they are lacking, particularly in the presence of ammonium, suggesting fewer or less active permeases, or a greater proportion subject to nitrogen repression (Kingsbury et al., 2004a, b; Nazi et al., 2007; Pascon et al., 2004). In addition, auxotrophs show defects in known cryptococcal virulence traits such as the ability to proliferate at $37{ }^{\circ} \mathrm{C}$, and melanin and capsule production (Kingsbury et al., 2004a, b; Pascon et al., 2004; Yang et al., 2002). We were therefore interested in evaluating the potential of threonine biosynthetic enzymes as anticryptococcal targets in C. neoformans. Several attempts to disrupt the homoserine and threonine biosynthetic gene HOM3 (encoding aspartate kinase, EC 2.7.2.4), and the threonine biosynthetic genes THR1 (encoding homoserine kinase, EC 2.7.1.39) and THR4 (encoding threonine synthase, EC 4.2.3.1) were unsuccessful. We demonstrate that this is because HOM3 and THR1, and likely other threonine biosynthetic enzymes, are essential for $C$. neoformans growth in most conditions.

\section{METHODS}

Strains, media and growth conditions. All S. cerevisiae strains used in this study (Table 1) were isogenic with S288c and C. neoformans 


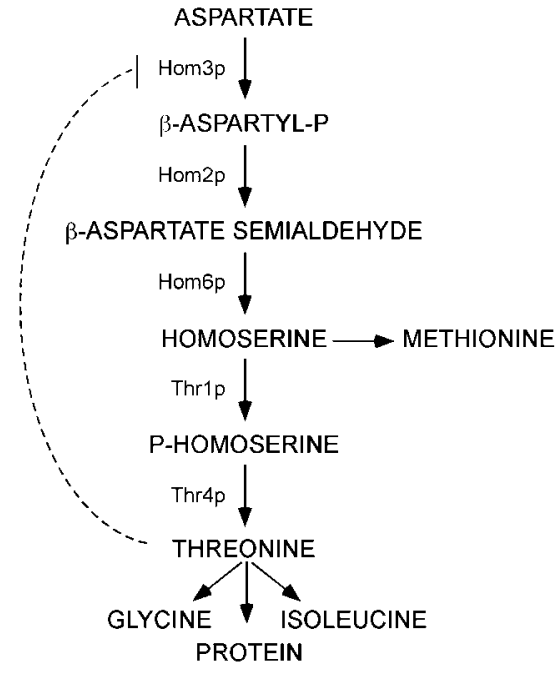

Fig. 1. Fungal threonine biosynthetic pathway.

strains were isogenic with H99 serotype A Mat $\alpha$, (Perfect et al., 1993). One Shot Top10 Chemically Competent Escherichia coli (Invitrogen) was used for plasmid propagation. Standard yeast and bacterial media were used (Sherman et al., 1974; Sambrook et al., 1989). Where specified, media were supplemented with nourseothricin (Nat; $100 \mu \mathrm{g}$ $\mathrm{ml}^{-1}$; Hans Knöll Institute für Naturstoff-Forschung, Jena, Germany), geneticin (200 $\mu \mathrm{g} \mathrm{ml}^{-1}$; Life Technologies), proline (1 g $\left.1^{-1}\right)$, sorbitol $(1 \mathrm{M})$, bathocuproinedisulfonic acid (BCS; $\left.200 \mu \mathrm{M}\right)$, cupric sulfate $\left(\mathrm{CuSO}_{4} ; 25 \mu \mathrm{M}\right)$, ascorbic acid $(1 \mathrm{mM})$, threonine $(2.5 \mathrm{mM})$, homoserine $(2.5 \mathrm{mM})$, methionine $(0.13 \mathrm{mM})$, Ala-Thr $(2.5 \mathrm{mM})$ and Met-Leu $(0.13 \mathrm{mM})$.

Identification of C. neoformans serotype A HOM3, THR1 and THR4. The predicted C. neoformans HOM3, THR1 and THR4 genes were initially identified from a C. neoformans serotype D strain JEC21 database that had been annotated by a genome-wide BLAST search (Loftus et al., 2005). NCBI accession numbers for the predicted Hom 3p, Thrlp and Thr4p included XP_572658, XP_572893 and XP_568789, respectively. Sequences were then BLASTed against the C. neoformans serotype A strain H99 sequence. Serotype A HOM3, THR1 and THR4 occurred in sequence with the NCBI accession numbers AACO02000077.1, AACO02000074.1 and AACO02000068.1, respectively.

We also attempted to isolate the C. neoformans HOM3, THR1 and THR4 cDNAs by complementation of the methionine and/or

Table 1. Strains used in this study

\begin{tabular}{|c|c|c|}
\hline Strain & Genotype & Source \\
\hline \multicolumn{3}{|c|}{ C. neoformans } \\
\hline $\mathrm{H} 99$ & Mat $\alpha$ & Perfect et al. (1993) \\
\hline $\mathrm{H} 99-73$ & NAT1-P ${ }_{C T R 4-1}-T H R 1$ & This study \\
\hline H99-76 & NAT1-P ${ }_{C T R 4-1}-H O M 3$ & This study \\
\hline \multicolumn{3}{|c|}{ S. cerevisiae } \\
\hline S157 & ura3s & Yang et al. (2002) \\
\hline S318 & ura3s thr $4 \Delta:$ : natMX4 & This study \\
\hline YJK1358 & ura3s thr1s:: kanMX4 & This study \\
\hline YJK2416 & ura3s hom $3 \Delta:$ : natMX4 & This study \\
\hline
\end{tabular}

threonine auxotrophies of $S$. cerevisiae hom $3 \Delta$, thr $1 \Delta$ and thr $4 \Delta$ strains, using a $C$. neoformans cDNA library. Specifically, $S$. cerevisiae

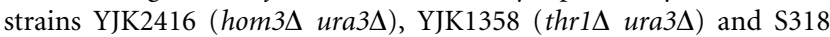
(thr4 4 ura3 $\Delta$ ) were transformed by lithium acetate-mediated transformation (Gietz et al., 1995), with a library that contained $C$. neoformans $\mathrm{H} 99$ cDNAs under the control of the S. cerevisiae GAL1 promoter in the pYES2.0 vector (Invitrogen) (Suvarna et al., 2000). $\mathrm{Ura}^{+}$transformants were screened for the acquisition of methionine and/or threonine prototrophy in the presence of galactose, but not glucose, as a carbon source. Plasmids that conferred prototrophy were isolated, propagated in E. coli $\mathrm{DH} 10 \mathrm{~B}$, then analysed by restriction analysis, and sequenced by the Duke University Cancer Center Sequencing Facility. Plasmids included pJO373 (pYES2.0 + C. neoformans THR1 cDNA, NCBI accession number EU623435), and pJO378 (pYES2.0+C. neoformans THR4 cDNA, NCBI accession number EU635873).

Plasmid and strain construction. In vitro $C$. neoformans thr4::NAT1, thr4::NEO, thr1::NAT1 and hom 3::NAT1 targeting cassettes were constructed using a modified PCR fusion technique (Davidson et al., 2002). To construct the thr4::NAT1 and thr4:: NEO targeting cassettes, the first rounds of PCR amplified 5' and $3^{\prime}$ THR4 sequence from H99 genomic DNA (primer pairs ZY125+JO257 and ZY126+JO255, respectively; see Supplementary Table S1 for sequences of all primers used in this study), and the NAT1 cassette from pGMC200 (McDade \& Cox, 2001) or the NEO cassette from pJAF1 (Fraser et al., 2003) (primers JO254+JO256). The gel-purified products were used as a template in the final fusion PCR with primers ZY125+ZY126. To construct the thr1::NAT1 targeting construct, the first round of PCR consisted of amplification of 5' and 3' THR1 sequence from H99 genomic DNA (primer pairs $\mathrm{JO} 298+\mathrm{JO} 303$ and $\mathrm{JO} 302+\mathrm{JO} 300$, respectively), and the NAT1 cassette from pGMC200 (primers JO301+JO304), then products were combined for the fusion PCR using primers JO298+JO300. Construction of the hom $3:$ NAT1 cassette consisted of amplification of $5^{\prime}$ and $3^{\prime} H O M 3$ sequence from $\mathrm{H} 99$ genomic DNA (primer pairs $\mathrm{JO} 318+\mathrm{JO} 316$ and JO320+JO315, respectively), and NAT1 from pGMC200 (primers JO314 + JO317), followed by fusion of products in the final PCR with primers JO318+JO320. All gel-purified constructs were cloned into pCR2.1-TOPO (Invitrogen) according to the manufacturer's instructions, resulting in pJO193 (thr4::NAT1), pJO221 (thr4::NEO), pJO249 (thr1::NAT1), and pJO260 (hom3:: NAT1).

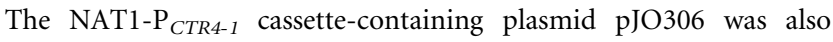
created by fusion PCR. The first round of PCR consisted of amplification of NAT1 from pGMC200 (primers JO357+JO412) and $\mathrm{P}_{\text {CTR4-1 }}$ from template pCTR4.2 (Ory et al., 2004) (primers JO408 + JO409). The purified products were used as a template in the final fusion PCR using primers JO357 + JO409, and the gel-purified final product was cloned into pCR2.1-TOPO.

In vitro targeting cassettes were constructed to place HOM3 and THR1 under control of the CTR4-1 promoter. To create the NAT1$\mathrm{P}_{\text {CTR4-1 }}$-HOM3 cassette, HOM3 upstream and the $5^{\prime}$ gene sequence were PCR-amplified from $\mathrm{H} 99$ genomic DNA (primer pairs $\mathrm{JO} 414+\mathrm{JO} 415$ and JO416+JO413), and NAT1-P ${ }_{\text {CTR4-1 }}$ was amplified from pJO306 (primers JO357+JO409). The products were combined in the fusion PCR (primers JO413+JO414), and the resulting product was gel-purified and cloned into pCR2.1-TOPO (pJO310). The first round of PCR for construction of the NAT1$\mathrm{P}_{\text {CTR4-1 }}$ THR1 cassette consisted of amplification of THR1 upstream and the $5^{\prime}$ gene sequence (primer pairs JO359+JO360 and

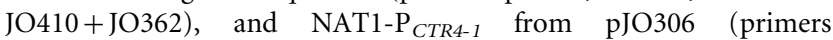
JO357 + JO409). The products were used as a template for the final fusion PCR using primers JO359+JO362, and the resulting product 
was cloned into pCR2.1-TOPO (pJO308). All plasmid constructions were confirmed by restriction digestion and PCR analyses.

The targeting cassettes were PCR-amplified from their respective plasmids, and introduced into strain $\mathrm{H} 99$ by biolistic transformation (Toffaletti et al., 1993). For transformation with the thr4::NAT1, thr4::NEO, thr1::NAT1, and hom $3:$ NAT1 constructs, transformation was performed on YPD + sorbitol, and after a $4 \mathrm{~h}$ incubation, cells were scraped off plates and spread on YPD + NAT or G418 plates to select for transformants. Transformants were purified and plated on SD to screen for acquisition of auxotrophy. For transformation

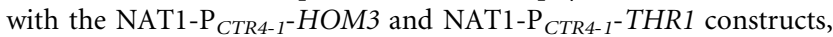
cells were plated on YPD + sorbitol + BCS and incubated for $2-3 \mathrm{~h}$ prior to transformation to allow for expression from $\mathrm{P}_{\text {CTR4. Following }}$ transformation, plates were incubated for $4 \mathrm{~h}$ and cells were replated on YPD + BCS + NAT. Purified transformants were screened for acquisition of auxotrophy on $\mathrm{SD}+\mathrm{CuSO}_{4}+$ ascorbic acid plates ( $\mathrm{P}_{\text {CTR4-1 }}$-repressing conditions). The NAT1- $\mathrm{P}_{\text {CTR } 4-1}$-THR1 genotype in strain H99-73 and NAT1-P CTR4-1 $^{-H O M 3}$ genotype in strain H9976 was confirmed by PCR (primer pairs JO281+JO300 and $\mathrm{JO} 506$ + JO280 for H99-73, and JO281 + JO320 and JO505+ JO280 for H99-76) and Southern hybridization analysis (Fig. 2).

The HOM3, THR1 and THR4 genes were replaced in the S. cerevisiae S157 strain by the natMX4 or kanMX4 cassettes, using PCR-mediated gene disruption (Goldstein \& McCusker, 1999; Wach et al., 1994).

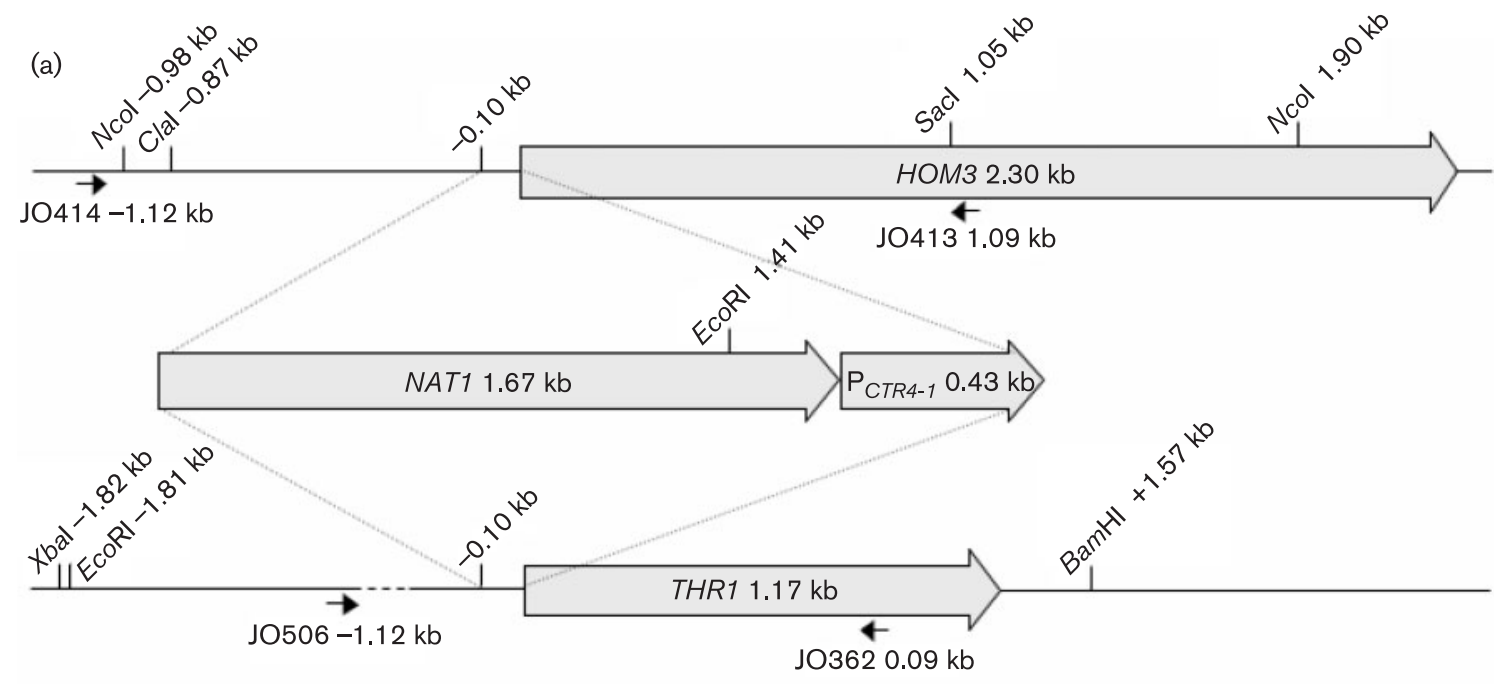

(b)
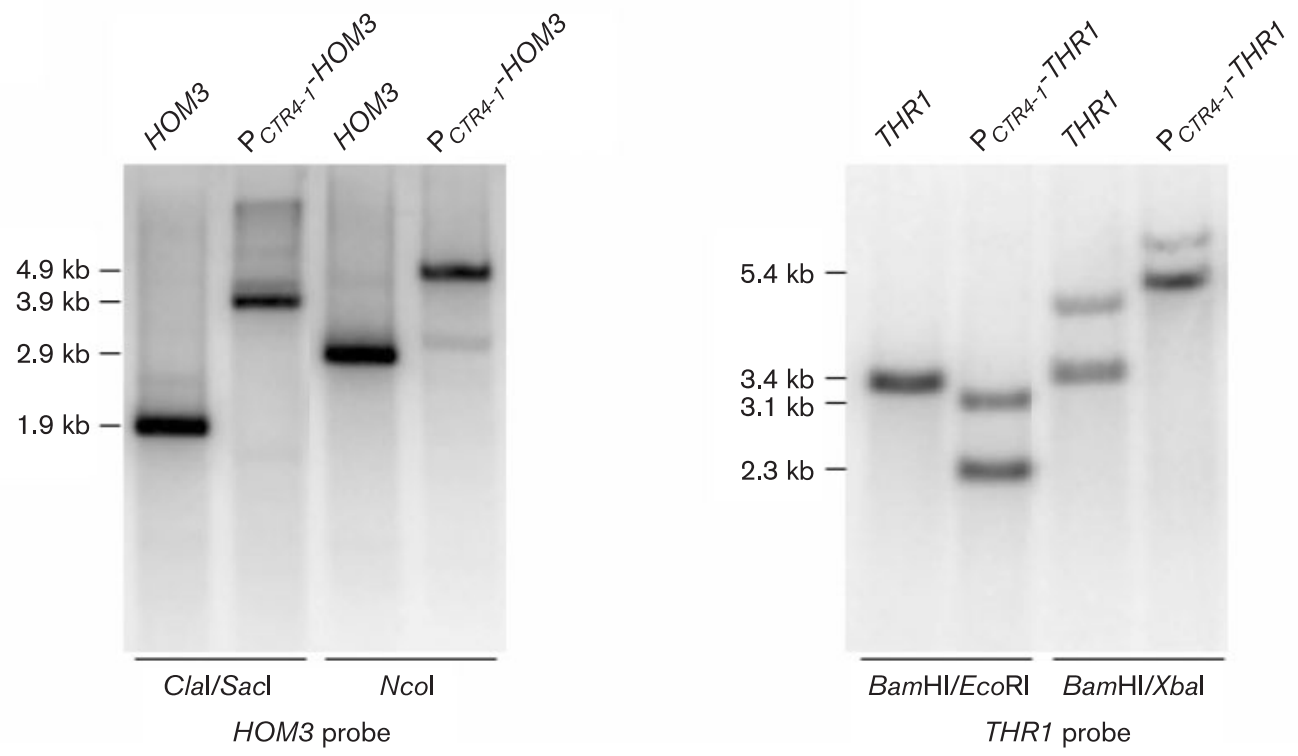

Fig. 2. (a) The HOM3 and THR1 genes were placed under the control of the $P_{C T R 4-1}$ promoter by inserting the NAT1-P $P_{\text {CTR4-1 }}$ construct immediately upstream of the predicted ORF, as shown in the diagram. (b) Southern hybridization analysis confirming correct positioning of the NAT1- $\mathrm{P}_{\text {CTR4-1 }}$ construct in strains H99-73 ( $\mathrm{P}_{\text {CTR4-1 }}-$ THR1) and H99-76 ( $\mathrm{P}_{\text {CTR4-1 }}$-HOM3). Genomic DNA from strains $\mathrm{H} 99$ (wild-type), $\mathrm{H} 99-73$ and $\mathrm{H} 99-76$ was digested with the restriction enzymes indicated, and blots were hybridized as indicated with a HOM3 or THR1 DNA probe, amplified using primer pairs J0414 + JO413 and JO506 + JO362, respectively. 
Gene deletions were confirmed by PCR, and by acquisition of methionine and/or threonine auxotrophy.

Manipulation of nucleic acids. Plasmid DNA from E. coli was extracted using the QIAprep Spin Miniprep kit (Qiagen), according to the manufacturer's instructions. Extraction of plasmid DNA from S. cerevisiae, and genomic DNA from C. neoformans for PCR analysis, was performed as described previously (Hoffman \& Winston, 1987). Genomic DNA from C. neoformans for Southern hybridization analysis was isolated as described previously (Yang et al., 2002), $2 \mu \mathrm{g}$ of which was digested with various restriction enzymes, separated by electrophoresis on a $0.75 \%(\mathrm{w} / \mathrm{v})$ agarose gel, denatured and transferred to a nylon membrane (Roche), as described previously (Sambrook et al., 1989).

RNA for Northern analyses was prepared from cells that had first been grown to a density of approximately $2 \times 10^{8}$ cells $\mathrm{ml}^{-1}$, in $50 \mathrm{ml}$ YPD + BCS. Cells were harvested, washed twice in sterile water, then split four ways and incubated with shaking in $50 \mathrm{ml}$ YPD + BCS or $\mathrm{YPD}+\mathrm{CuSO}_{4}+$ ascorbic acid, at $25{ }^{\circ} \mathrm{C}$ or $37{ }^{\circ} \mathrm{C}$. Following incubation for $5 \mathrm{~h}$, RNA was isolated as described previously (Yang et al., 2002). Each sample was prepared in duplicate, and $10 \mu \mathrm{g}$ duplicates of each preparation were separated in a $1 \%(\mathrm{w} / \mathrm{v})$ agarose-formaldehyde gel, and transferred to a nylon membrane.

Probes for Southern and Northern hybridizations were prepared from gel-purified PCR products. Specifically, probes for Southern hybridizations were amplified using primer pairs JO413 + JO414 (HOM3) and JO362+JO506 (THR1). Primer pairs for amplification of Northern hybridization probes included JO770 + JO772 (HOM3), $\mathrm{JO} 298$ + JO362 (THR1), JO223 + JO225 (GPD) and JO765 + JO766 (CTR4) (see Table S1 for primer sequences). Probes were labelled with $\left[\alpha_{-}{ }^{32} \mathrm{P}\right] \mathrm{dCTP}$ (Perkin-Elmer) using the RediprimeII Random Prime Labelling System (Amersham Biosciences), according to the manufacturer's instructions. Blots were prehybridized and hybridized in ULTRAhyb buffer (Ambion), and washed according to the manufacturer's instructions. Membrane signal was visualized using a Typhoon 9200 Variable Mode Imager (Molecular Dynamics), and band signal intensity was quantified using ImageQuaNT 5.2 software (Molecular Dynamics).

\section{RESULTS AND DISCUSSION}

\section{Identification of HOM3, THR1 and THR4 in C. neoformans}

Given the absence of the threonine biosynthetic pathway in humans (Payne \& Loomis, 2006) and the avirulence or inability to survive in vivo of various amino acid auxotrophs, we were interested in assessing the potential of threonine biosynthetic enzymes as antifungal drug targets in the human-pathogenic fungus $C$. neoformans. In particular, we focused on, first, the aspartate kinase (encoded by HOM3), the initial and key feedback regulatory enzyme of the pathway. The avirulence of other cryptococcal methionine auxotrophs (Nazi et al., 2007; Pascon et al., 2004; Yang et al., 2002) indicates that $C$. neoformans is unable to supplement this auxotrophy in the in vivo environment, thus we reasoned that the combined threonine and methionine auxotrophies of hom 3 mutants should be even more detrimental to survival in vivo and/or virulence. Moreover, HOM3 is required for the in vivo survival of S. cerevisiae (Kingsbury et al., 2006). We were also interested in the final two steps of threonine biosynthesis, catalysed by homoserine kinase (encoded by THR1) and threonine synthase (encoded by THR4). Mutation of these genes in S. cerevisiae results in a plethora of deleterious phenotypes in addition to auxotrophy (Birrell et al., 2001, 2002; Care et al., 2004; Deutschbauer et al., 2002; Dunn et al., 2006; Enyenihi \& Saunders, 2003; Giaever et al., 2002; Roberg et al., 1997), which may also influence in vivo survival and/or virulence.

We identified the putative C. neoformans H99 HOM3, THR1 and THR4 genes from the C. neoformans serotype A strain $\mathrm{H} 99$ through sequence similarity with the respective predicted ORFs in serotype D. Furthermore, cDNAs matching the predicted THR1 and THR4 genes were isolated from a C. neoformans H99 cDNA library based on the ability to confer threonine prototrophy to S. cerevisiae thr $1 \Delta$ and thr $4 \Delta$ strains, respectively (Fig. 3), thus verifying that the identified genes encoded the predicted enzyme activities. We were unable to isolate the HOM3 cDNA by complementation of a S. cerevisiae hom $3 \Delta$ strain, however, likely due to under-representation of the HOM3 cDNA in the library. Consistent with this, we were unable to PCRamplify the HOM3 cDNA from the library DNA. The $C$. neoformans H99 HOM3, THR1 and THR4 genes were predicted to contain six, two and five introns, respectively. The predicted C. neoformans Hom 3p, Thrlp and Thr4p sequences were highly similar to the corresponding

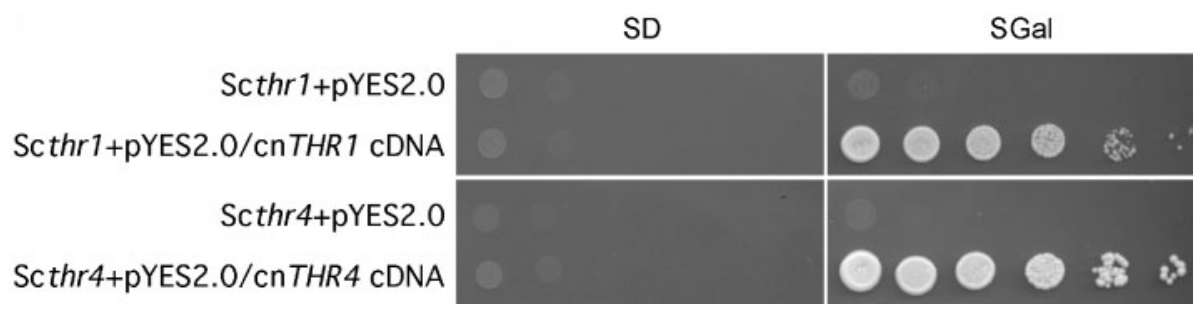

Fig. 3. Functional complementation of $S$. cerevisiae thr1 (YJK1358) and thr4 (S318) strains by C. neoformans THR1 and THR4 cDNA. Tenfold dilutions of YJK1358 transformed with pYES2.0 or pJO373 (pYES2.0/cnTHR1 cDNA), and S318 transformed with pYES2.0 or pJO378 (pYES2.0/cnTHR4 cDNA), were plated on SD (synthetic dextrose) or synthetic galactose (SGal) medium, and incubated at $30{ }^{\circ} \mathrm{C}$ for 3 days. 
proteins in S. cerevisiae, with approximately 50, 56 and $50 \%$ amino acid identity, respectively.

\section{C. neoformans HOM3, THR1 and THR4 are recalcitrant to disruption}

In order to study the phenotypes of C. neoformans hom3, thr1 and thr4 mutants, we attempted to disrupt HOM3, THR1 and THR4 in the serotype A strain H99, using hom $3:$ :NAT1, thr1::NAT1, thr4::NAT1 and thr4::NEO targeting cassettes. However, no auxotrophic mutants were obtained after screening 151 transformants for HOM3 disruption, 89 transformants for THR1 disruption, and 469 transformants for THR4 disruption. An inability to disrupt these genes may be due to HOM3, THR1 and THR4 being essential for $C$. neoformans growth.

\section{C. neoformans HOM3 and THR1 are essential}

To determine whether threonine biosynthetic genes are essential in C. neoformans, we replaced the endogenous promoters of HOM3 and THR1 with the $\mathrm{P}_{\text {CTR4-1 }}$ promoter (Ory et al., 2004), thereby placing the genes under copperrepressible control (Fig. 2). Growth of the wild-type (H99), $\mathrm{P}_{\text {CTR4-1-HOM3 (H99-76) and } \mathrm{P}_{\text {CTR4-1 }} \text {-THR1 (H99-73) }}$ strains was compared by plating 10 -fold spot dilutions of strains, pre-grown in YPD $+\mathrm{BCS}$, onto YPD $+\mathrm{BCS}$ (promoter-inducing conditions) and $\mathrm{YPD}+\mathrm{CuSO}_{4}+$ ascorbic acid (promoter-repressing conditions) (Fig. 4). After incubation at $30{ }^{\circ} \mathrm{C}$ for 3 days in promoter-inducing conditions, $\mathrm{P}_{\text {CTR4-1 }}$-HOM3 and $\mathrm{P}_{\text {CTR4-1 }}$-THR1 strains grew considerably, although less well than the wild-type as judged by colony size, indicating that HOM3 and THR1 were expressed at different from normal level in these strains. However, no colony formation was observed for the $\mathrm{P}_{C T R 4-1^{-}}$ HOM3 and $\mathrm{P}_{\text {CTR4-1 }}$-THR1 strains in promoter-repressing conditions. Since the yeast extract and Bacto peptone in
YPD contains significant levels of threonine and methionine for supplementation of Thr and Met auxotrophies (Difco Manual, 11th edition), these results indicate that THR1 and HOM3 are essential in C. neoformans, even in the presence of abundant threonine and methionine.

\section{The essential phenotype is dependent on nitrogen source and amino acid form}

The lack of growth of the $\mathrm{P}_{C T R 4-1}$-HOM3 strain on rich medium (YPD) in promoter-repressing conditions is likely not due to an inability of $C$. neoformans to supplement the methionine auxotrophy of this strain, since $C$. neoformans met2, met 3 and met 6 mutants all grow on YPD, and their methionine auxotrophy can be supplemented by methionine or methionine dipeptides, when either ammonium or proline is the nitrogen source (Nazi et al., 2007; Pascon et al., 2004; Yang et al., 2002). Since both $\mathrm{P}_{C T R 4-1}-H O M 3$ and $\mathrm{P}_{\text {CTR4-1 }}$-THR1 strains were unable to grow under promoter-repressing conditions, the growth deficiency on YPD may instead be due to $C$. neoformans being unable to satisfy the threonine auxotrophy in this medium.

Growth of the $\mathrm{P}_{C T R 4-1}-$ HOM 3 and $\mathrm{P}_{\text {CTR 4-1 }}$-THR1 strains was compared by spot dilutions on minimal medium containing ammonium (SD) or proline [SD(Pro)] as the nitrogen source, supplemented with various combinations of homoserine, methionine and threonine amino acids or dipeptides, in $\mathrm{P}_{C T R 4-1}$-repression conditions (Fig. 5). While no colony formation of strains was observed in $\mathrm{P}_{C T R 4-1^{-}}$ repression conditions in the absence of amino acid supplements, residual growth similar to that present on YPD in repressing conditions was observed, which we attribute to a basal level of gene expression still occurring, and/or utilization of cell reserves accumulated during the pre-growth in gene-expressing conditions. Strains grew no better when the amino acids methionine and threonine were added to either $\mathrm{SD}$ or $\mathrm{SD}$ (Pro) medium than on

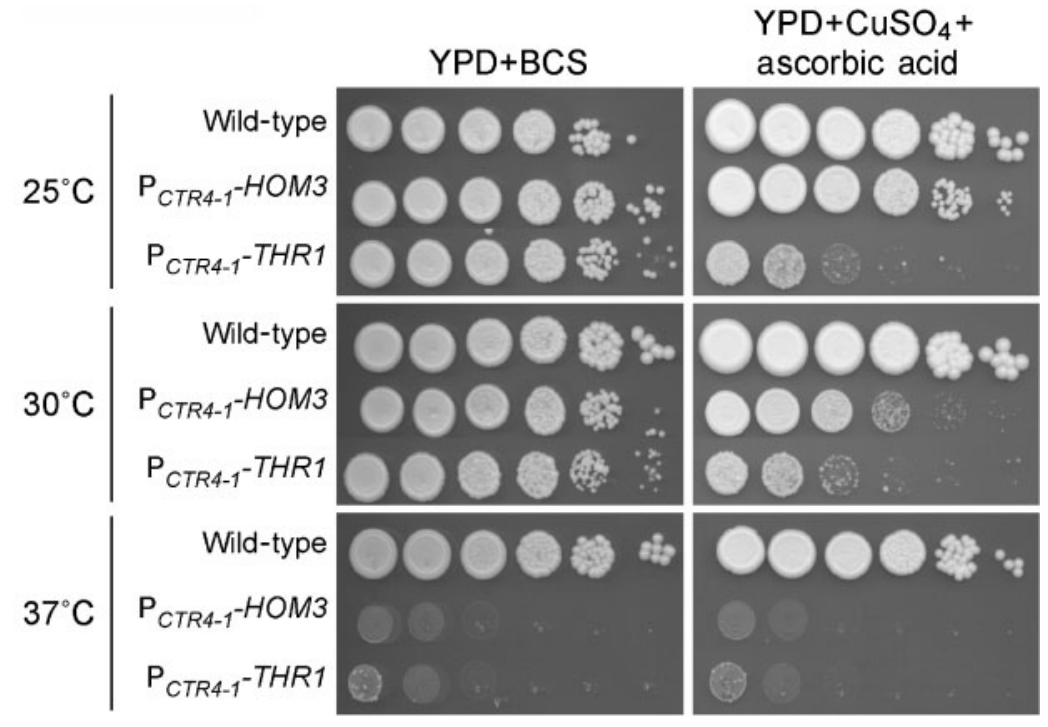

Fig. 4. Temperature-dependent phenotypes of $\mathrm{P}_{\text {CTR } 4-1}-\mathrm{HOM} 3$ (H99-76), $\mathrm{P}_{\text {CTR4-1 }}$-THR1 (H99-73) and the wild-type (H99) strains in gene-repressing ( $\mathrm{CuSO}_{4}+$ ascorbic acid) and gene-inducing (BCS) conditions. Tenfold dilutions of strains were plated and incubated for 3 days. 


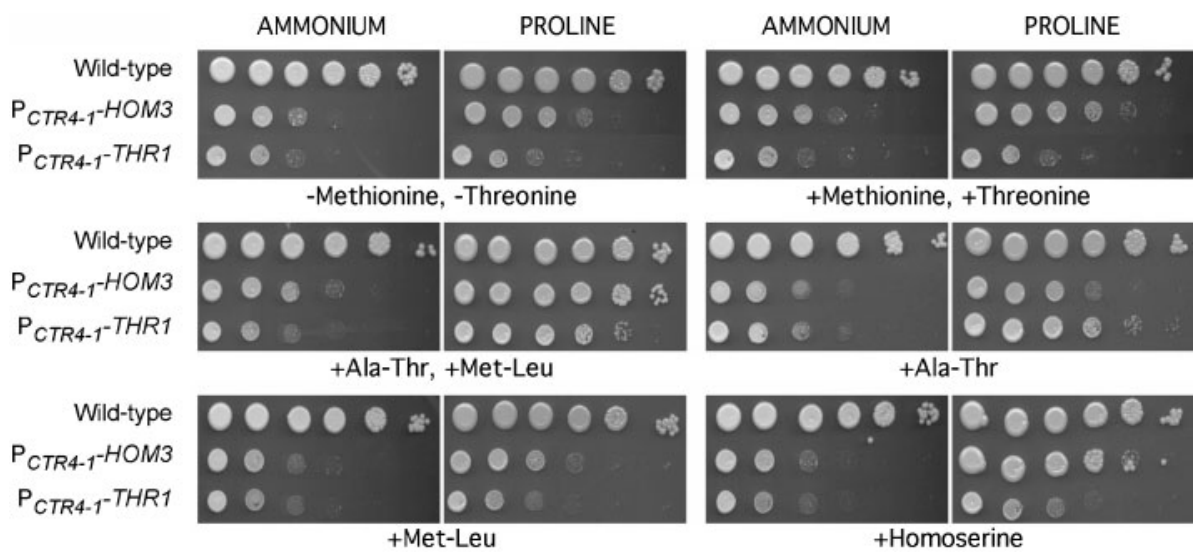

Fig. 5. Auxotrophic supplementation of $P_{C T R 4-1}-H O M 3(H 99-76)$ and $P_{C T R 4-1}-T H R 1(H 99-73)$ strains. Strains were serially diluted tenfold, and plated on $\mathrm{SD}+\mathrm{CuSO}_{4}+$ ascorbic acid or $\mathrm{SD}(\mathrm{Pro})+\mathrm{CuSO}_{4}+$ ascorbic acid that was supplemented with various combinations of methionine, threonine, Ala-Thr, Met-Leu or homoserine, as indicated. Plates were incubated for 3 days at $30{ }^{\circ} \mathrm{C}$.

media lacking amino acids. Strains were also unable to grow when SD medium was supplemented with threonine and methionine dipeptides, but growth was enhanced above background levels when the threonine and methionine dipeptides were added to $\mathrm{SD}$ (Pro) medium. Furthermore, growth of the $\mathrm{P}_{\text {CTR4-1 }}$-THR1 strain required only threonine dipeptides, while growth of the $\mathrm{P}_{\text {CTR4-1 }}{ }^{-}$ HOM3 strain required both methionine and threonine dipeptides, or homoserine, providing further evidence that these genes indeed confer homoserine kinase and aspartate kinase activities, respectively.

The demonstration that threonine dipeptides, but not amino acids, supplement cryptococcal threonine auxotrophy, and only in the presence of proline, but not ammonium, as a sole nitrogen source, is similar to results observed previously for supplementation of other $C$. neoformans auxotrophies (Kingsbury et al., 2004a, b). Combined, these results indicate that $C$. neoformans possesses fewer amino acid permeases, permeases that have a lower transport velocity, or more permeases that are subject to ammonium repression. Although YPD medium contains significant levels of peptides, our results indicate that the lack of growth in this medium is likely due to nitrogen repression, and/or transport of other peptides outcompeting transport of threonine-containing peptides.

\section{Temperature determines severity of phenotype}

While the inability of a cryptococcal amino acid auxotroph to grow on YPD at $30{ }^{\circ} \mathrm{C}$ has not been previously demonstrated, it has been shown that SPE3-lys9 (lysine auxotrophic) and ilv2 (isoleucine and valine auxotrophic) mutants die in YPD at $37{ }^{\circ} \mathrm{C}$ (Kingsbury et al., 2004a, b). We therefore determined whether the incubation temperature affects growth of $\mathrm{P}_{\text {CTR } 4-1}-H O M 3$ and $\mathrm{P}_{\text {CTR4-1 }}$-THR1 strains on YPD. Interestingly, reduction of the incubation temperature to $25{ }^{\circ} \mathrm{C}$ allowed the growth of the $\mathrm{P}_{\text {CTR4-1 }}{ }^{-}$ HOM3 strain in repressing conditions, while the $\mathrm{P}_{\text {CTR4-1 }}{ }^{-}$ THR1 strain was no better able to grow at this temperature than at $30{ }^{\circ} \mathrm{C}$ (Fig. 4).

Differences in the growth phenotype between the two strains could be attributable to either homoserine kinase having a role in addition to threonine biosynthesis in $C$. neoformans, or the block in the biosynthetic pathway caused by inhibition of homoserine kinase leading to the accumulation of a toxic intermediate. Consistent with this, elevated levels of the intermediate predicted to accumulate, homoserine, are toxic to mammalian (Rees et al., 1994) and bacterial cells (Kotre et al., 1973; O'Barr \& Everett, 1971). Moreover, the accumulation of a toxic intermediate has been hypothesized to be responsible for toxic effects associated with other amino acid biosynthetic mutants (Arevalo-Rodriguez et al., 2004; Kingsbury et al., 2004a; Pascon et al., 2004; Suliman et al., 2007), and may explain deleterious phenotypes associated with THR1 and THR4 mutation in S. cerevisiae. In S. cerevisiae, the threonine pathway is regulated positively in response to threonine starvation, by upregulation of gene transcription and eliminating feedback inhibition (Hinnebusch, 1992; Martin-Rendon et al., 1993; Mountain et al., 1991; Ramos \& Calderon, 1992). If the pathway is similarly regulated in $C$. neoformans, threonine starvation conditions, such as what Thrlp-inhibited C. neoformans would likely encounter in vivo, should result in increased flux through the threonine biosynthetic pathway, thus increased toxic intermediate accumulation, and hence intensified growth defects.

We also compared the growth of $\mathrm{P}_{\text {CTR4-1 }}$ HOM3 and $\mathrm{P}_{\text {CTR4-1 }}$-THR1 strains at $37^{\circ} \mathrm{C}$. While some background growth of both strains was still present at $30^{\circ} \mathrm{C}$ in repressing conditions, growth was completely eliminated at 

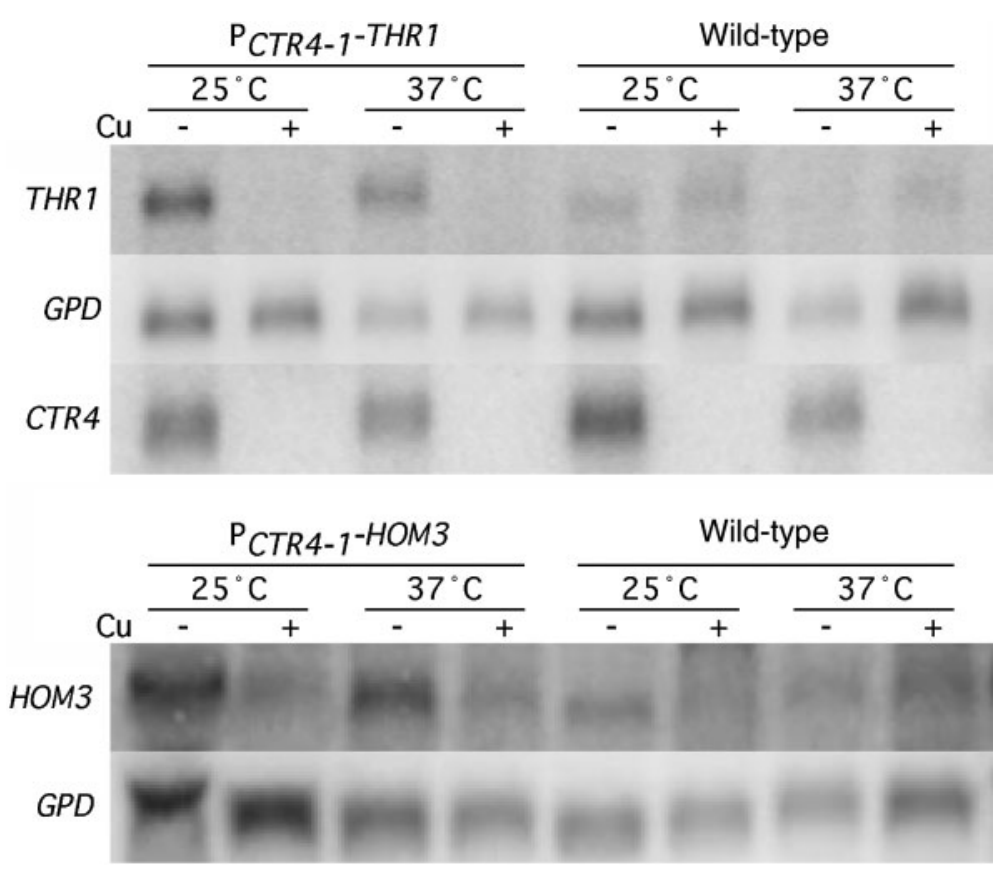

Fig. 6. Northern hybridization analysis comparing THR1, HOM3, CTR4 and GPD transcript abundance. RNA was prepared from H99 (wild-type), H99-73 ( $\mathrm{P}_{\text {CTR4-1 }}-$ THR1), and H99-76 ( $\mathrm{P}_{\mathrm{CTR} 4-1}-\mathrm{HOM3}$ ) strains, grown in $\mathrm{P}_{\mathrm{CTR} 4-1}$-repressing (YPD + $\mathrm{CuSO}_{4}+$ ascorbic acid; + $\mathrm{Cu}$ ) or $\mathrm{P}_{\mathrm{CTR} 4-1}$-inducing (YPD + BCS; $-\mathrm{Cu}$ ) conditions, at $25^{\circ} \mathrm{C}$ or $37^{\circ} \mathrm{C}$.
$37{ }^{\circ} \mathrm{C}$ in repressing conditions. Surprisingly, growth was also eliminated in induction conditions, which may indicate that there is a greater difference between expression from the CTR4-1 promoter and endogenous THR1 and HOM3 expression at $37{ }^{\circ} \mathrm{C}$, compared with $30{ }^{\circ} \mathrm{C}$. To examine this, we compared HOM3, THR1 and CTR4 transcript levels following a $5 \mathrm{~h}$ incubation of the $\mathrm{P}_{\text {CTR4-1 }}$-HOM3, $\mathrm{P}_{\text {CTR4-1 }}$-THR1 and wild-type strains, in promoter-induction and repression conditions, at 25 and $37{ }^{\circ} \mathrm{C}$ (Fig. 6). Following normalization to the GPD housekeeping gene, we observed no obvious temperaturedependent changes in THR1, HOM3 or CTR4 transcript levels from the $\mathrm{P}_{C T R 4-1}-H O M 3, \mathrm{P}_{C T R 4-1}-$ THR1 and wildtype strains, respectively, grown in promoter-induction conditions; thus the CTR4 (and CTR4-1) promoter is not regulated by temperature. Furthermore, although HOM3 transcript levels were barely detectable at both temperatures, THR1 and HOM3 transcription levels in the wildtype did not appear to be enhanced at $37{ }^{\circ} \mathrm{C}$ compared with $25{ }^{\circ} \mathrm{C}$. Results also show that HOM3 and THR1 transcripts expressed from $\mathrm{P}_{\text {CTR4-1- }}$ HOM3 and $\mathrm{P}_{\text {CTR4-1 }}{ }^{-}$ THR1 strains in induction conditions were at higher than wild-type levels at both 30 and $37{ }^{\circ} \mathrm{C}$. One possible explanation for the lack of growth in induction conditions is that higher expression of these genes might result in growth impairment by perturbing metabolic flux, interfering with general cell metabolism, which may be accentuated at higher temperatures. Given our SPE3-lys 9 and ilv2 findings, the increased severity of growth defects at $37{ }^{\circ} \mathrm{C}$ in repressing conditions may be due to decreased threonine transport at this temperature.

Given the different niche occupation and evolutionary distance between $S$. cerevisiae, an ascomycete, and $C$. neoformans, a basidiomycete, it is not surprising to see differences in gene requirement between species; for example, the fatty acid synthesis genes FAS1 and FAS2, and $R A M 1$ required for signalling, are essential in $C$. neoformans but not in S. cerevisiae and/or Candida albicans (Chayakulkeeree et al., 2007; Vallim et al., 2004). However, given the highly conserved nature of the threonine biosynthetic pathway between fungi, it is surprising to find that threonine biosynthetic genes are essential in $C$. neoformans, and to our knowledge, this is the first documented case of threonine biosynthetic gene necessity in fungi. The essential nature of these genes, particularly at $37{ }^{\circ} \mathrm{C}$, makes aspartate kinase and homoserine kinase excellent candidates for anti-cryptococcal drug targets.

\section{ACKNOWLEDGEMENTS}

The authors would like to thank Dr Zhonghui Yang for some strain construction, Dr Joseph Heitman for comments on the manuscript, and Drs James Fraser and Tamara Doering for the generous gifts of plasmids pJAF1 and pCTR4-2, respectively. We also thank Dr Brian Wong for the C. neoformans cDNA library, which was a gift to the Duke University Mycology Research Unit. This study was funded by the National Institutes of Health R01 grant GM070541 and R21 grant AI070247.

\section{REFERENCES}

Arevalo-Rodriguez, M., Pan, X., Boeke, J. D. \& Heitman, J. (2004). FKBP12 controls aspartate pathway flux in Saccharomyces cerevisiae to prevent toxic intermediate accumulation. Eukaryot Cell 3, 1287-1296.

Birrell, G. W., Giaever, G., Chu, A. M., Davis, R. W. \& Brown, J. M. (2001). A genome-wide screen in Saccharomyces cerevisiae for genes affecting UV radiation sensitivity. Proc Natl Acad Sci U S A 98, 1260812613. 
Birrell, G. W., Brown, J. A., Wu, H. I., Giaever, G., Chu, A. M., Davis, R. W. \& Brown, J. M. (2002). Transcriptional response of Saccharomyces cerevisiae to DNA-damaging agents does not identify the genes that protect against these agents. Proc Natl Acad Sci U S A 99, 8778-8783.

Care, A., Vousden, K. A., Binley, K. M., Radcliffe, P., Trevethick, J., Mannazzu, I. \& Sudbery, P. E. (2004). A synthetic lethal screen identifies a role for the cortical actin patch/endocytosis complex in the response to nutrient deprivation in Saccharomyces cerevisiae. Genetics 166, 707-719.

Chayakulkeeree, M., Rude, T. H., Toffaletti, D. L. \& Perfect, J. R. (2007). Fatty acid synthesis is essential for survival of Cryptococcus neoformans and a potential fungicidal target. Antimicrob Agents Chemother 51, 3537-3545.

Davidson, R. C., Blankenship, J. R., Kraus, P. R., de Jesus Berrios, M., Hull, C. M., D'Souza, C., Wang, P. \& Heitman, J. (2002). A PCR-based strategy to generate integrative targeting alleles with large regions of homology. Microbiology 148, 2607-2615.

Deutschbauer, A. M., Williams, R. M., Chu, A. M. \& Davis, R. W. (2002). Parallel phenotypic analysis of sporulation and postgermination growth in Saccharomyces cerevisiae. Proc Natl Acad Sci U S A 99, 15530-15535.

Dunn, C. D., Lee, M. S., Spencer, F. A. \& Jensen, R. E. (2006). A genomewide screen for petite-negative yeast strains yields a new subunit of the i-AAA protease complex. Mol Biol Cell 17, 213-226.

Enyenihi, A. H. \& Saunders, W. S. (2003). Large-scale functional genomic analysis of sporulation and meiosis in Saccharomyces cerevisiae. Genetics 163, 47-54.

Fraser, J. A., Subaran, R. L., Nichols, C. B. \& Heitman, J. (2003). Recapitulation of the sexual cycle of the primary fungal pathogen Cryptococcus neoformans var. gattii: implications for an outbreak on Vancouver Island, Canada. Eukaryot Cell 2, 1036-1045.

Giaever, G., Chu, A. M., Ni, L., Connelly, C., Riles, L., Véronneau, S., Dow, S., Lucau-Danila, A., Anderson, K. \& other authors (2002). Functional profiling of the Saccharomyces cerevisiae genome. Nature 418, 387-391.

Gietz, R. D., Schiestl, R. H., Willems, A. R. \& Woods, R. A. (1995). Studies on the transformation of intact yeast cells by the LiAc/SSDNA/PEG procedure. Yeast 11, 355-360.

Goldstein, A. L. \& McCusker, J. H. (1999). Three new dominant drug resistance cassettes for gene disruption in Saccharomyces cerevisiae. Yeast 15, 1541-1553.

Hinnebusch, A. (1992). General and pathway-specific regulatory mechanisms controlling the synthesis of amino acid biosynthetic enzymes in Saccharomyces cerevisiae. In The Molecular and Cellular Biology of the Yeast Saccharomyces: Gene Expression, pp. 319-414. Edited by E. W. Jones, J. R. Pringle \&. J. R. Broach. Cold Spring Harbor, NY: Cold Spring Harbor Laboratory Press.

Hoffman, C. S. \& Winston, F. (1987). A ten-minute DNA preparation from yeast efficiently releases autonomous plasmids for transformation of Escherichia coli. Gene 57, 267-272.

Jones, E. W. \& Fink, G. R. (1982). Regulation of amino acid and nucleotide biosynthesis in yeast. In The Molecular Biology of the Yeast Saccharomyces: Metabolism and Gene Expression. Edited by J. N. Strathern, E. W. Jones \& J. R. Broach. Cold Spring Harbor, NY: Cold Spring Harbor Laboratory.

Kingsbury, J. M., Yang, Z., Ganous, T. M., Cox, G. M. \& McCusker, J. H. (2004a). Cryptococcus neoformans Ilv2p confers resistance to sulfometuron methyl and is required for survival at $37^{\circ} \mathrm{C}$ and in vivo. Microbiology 150, 1547-1558.

Kingsbury, J. M., Yang, Z., Ganous, T. M., Cox, G. M. \& McCusker, J. H. (2004b). A novel chimeric spermidine synthase-saccharopine dehydrogenase (SPE3-LYS9) gene in the human pathogen Cryptococcus neoformans. Eukaryot Cell 3, 752-763.

Kingsbury, J. M., Goldstein, A. L. \& McCusker, J. H. (2006). Role of nitrogen and carbon transport, regulation, and metabolism genes for Saccharomyces cerevisiae survival in vivo. Eukaryot Cell 5, 816-824.

Kotre, A. M., Sullivan, S. J. \& Savageau, M. A. (1973). Metabolic regulation by homoserine in Escherichia coli B-r. J Bacteriol 116, 663-672.

Loftus, B. J., Fung, E., Roncaglia, P., Rowley, D., Amedeo, P., Bruno, D., Vamathevan, J., Miranda, M., Anderson, I. J. \& other authors (2005). The genome of the basidiomycetous yeast and human pathogen Cryptococcus neoformans. Science 307, 1321-1324.

Martin-Rendon, E., Farfan, M. J., Ramos, C. \& Calderon, I. L. (1993). Isolation of a mutant allele that deregulates the threonine biosynthesis in Saccharomyces cerevisiae. Curr Genet 24, 465-471.

McDade, H. C. \& Cox, G. M. (2001). A new dominant selectable marker for use in Cryptococcus neoformans. Med Mycol 39, 151-154.

Mountain, H. A., Bystrom, A. S., Larsen, J. T. \& Korch, C. (1991). Four major transcriptional responses in the methionine/threonine biosynthetic pathway of Saccharomyces cerevisiae. Yeast 7, 781-803.

Nazi, I., Scott, A., Sham, A., Rossi, L., Williamson, P. R., Kronstad, J. W. \& Wright, G. D. (2007). Role of homoserine transacetylase as a new target for antifungal agents. Antimicrob Agents Chemother 51, 1731-1736.

O'Barr, T. P. \& Everett, K. A. (1971). Effect of L-homoserine on the growth of Mycobacterium tuberculosis. Infect Immun 3, 328-332.

Ory, J. J., Griffith, C. L. \& Doering, T. L. (2004). An efficiently regulated promoter system for Cryptococcus neoformans utilizing the CTR4 promoter. Yeast 21, 919-926.

Pascon, R. C., Ganous, T. M., Kingsbury, J. M., Cox, G. M. \& McCusker, J. H. (2004). Cryptococcus neoformans methionine synthase: expression analysis and requirement for virulence. Microbiology 150, 3013-3023.

Payne, S. H. \& Loomis, W. F. (2006). Retention and loss of amino acid biosynthetic pathways based on analysis of whole-genome sequences. Eukaryot Cell 5, 272-276.

Perfect, J. R., Toffaletti, D. L. \& Rude, T. H. (1993). The gene encoding phosphoribosylaminoimidazole carboxylase (ADE2) is essential for growth of Cryptococcus neoformans in cerebrospinal fluid. Infect Immun 61, 4446-4451.

Ramos, C. \& Calderon, I. L. (1992). Overproduction of threonine by Saccharomyces cerevisiae mutants resistant to hydroxynorvaline. Appl Environ Microbiol 58, 1677-1682.

Rees, W. D., Grant, S. D., Hay, S. M. \& Saqib, K. M. (1994). Threonine synthesis from homoserine as a selectable marker in mammalian cells. Biochem J 299, 637-644.

Roberg, K. J., Bickel, S., Rowley, N. \& Kaiser, C. A. (1997). Control of amino acid permease sorting in the late secretory pathway of Saccharomyces cerevisiae by Sec13, Lst4, Lst7 and Lst8. Genetics 147, 1569-1584.

Sambrook, J., Fritsch, E. F. \& Maniatis, T. (1989). Molecular Cloning: a Laboratory Manual. Cold Spring Harbor, NY: Cold Spring Harbor Laboratory.

Sherman, F., Fink, G. R. \& Lawrence, C. W. (1974). Methods in Yeast Genetics. Cold Spring Harbor, NY: Cold Spring Harbor Laboratory.

Suliman, H. S., Appling, D. R. \& Robertus, J. D. (2007). The gene for cobalamin-independent methionine synthase is essential in Candida albicans: a potential antifungal target. Arch Biochem Biophys 467, 218 226.

Suvarna, K., Bartiss, A. \& Wong, B. (2000). Mannitol-1-phosphate dehydrogenase from Cryptococcus neoformans is a zinc-containing 
long-chain alcohol/polyol dehydrogenase. Microbiology 146, 27052713.

Toffaletti, D. L., Rude, T. H., Johnston, S. A., Durack, D. T. \& Perfect, J. R. (1993). Gene transfer in Cryptococcus neoformans by use of biolistic delivery of DNA. J Bacteriol 175, 1405-1411.

Vallim, M. A., Fernandes, L. \& Alspaugh, J. A. (2004). The RAM1 gene encoding a protein-farnesyltransferase $\beta$-subunit homologue is essential in Cryptococcus neoformans. Microbiology 150, 1925-1935.
Wach, A., Brachat, A., Pohlmann, R. \& Philippsen, P. (1994). New heterologous modules for classical or PCR-based gene disruptions in Saccharomyces cerevisiae. Yeast 10, 1793-1808.

Yang, Z., Pascon, R. C., Alspaugh, A., Cox, G. M. \& McCusker, J. H. (2002). Molecular and genetic analysis of the Cryptococcus neoformans MET3 gene and a met3 mutant. Microbiology 148, 2617-2625.

Edited by: J. F. Ernst 